encoding these molecules have been cloned. With rigorous linkage studies and a host of candidate genes, the molecular defects leading to RP and other forms of RP are likely to be revealed in near future. Research Committee on Chorioretinal Degenerations, The Ministry of Health and Welfare of Japan, has also doing research since 1977 to study the causes and the possibility of therapy, and to establish clinical and etiological diagnosis including DNA of RP.

\title{
SYMPOSIUM II. DNA Analysis
}

SII-1. ORNITHINE TRANSCARBAMYLASE DEFICIENCY-PHENOTYFE TO GENOTYPE. Ichiro MATSUDA (Dept. Pediatr., Kumamoto Univ. Med. Sch., Kumamoto $860)$

The incidence of the five major disorders of the urea cycle was estimated to be 1 in 48,000 live births in Japan; The incidence of ornithine transcarbamylase (OTC) deficiency (X-linked inheritance) was the highest, the rate being 1 in 80,000 live births. We did a retrospective study of urea cycle disorders we encountered clinically between 1978-88. The 32 male patients found to have the disease were classified into two groups depending on age-at-onset; neonatal-onset group (0-28D) and late-onset group (>28D). In the first group, 2 patients with $0 \%$ of OTC had a nonsense $\mathrm{C} \rightarrow \mathrm{T}$ mutation in exon 5 , and one patient with $8 \%$ of OTC had a missense $A \rightarrow T$ mutation in exon 6 (Val-Asp). In the second group, 2 patients with $20 \%$ of OTC had a missense $\mathrm{C} \rightarrow \mathrm{T}$ mutation in exon 8 (Arg-Tryp). The relationship between genotypes and phenotypes in OTC deficiency is the subject of on going study.

SII-2. DNA ANALYSIS ON CHROMOSOME MICRODELETION SYNDROMES: APPROACH TO UNDERSTANDING THE CAUSES OF PRADER-WILLI (PWS), ANGELMAN (AS), AND LANGER-GIEDION (LGS) SYNDROMES. Norio NIIKAWA (Dept. Hum. Genet., Nagasaki Univ. Sch. Med., Nagasaki 852)

PWS, AS and LGS are considered as contiguous gene syndromes, since they are often associated with chromosome microdeletions and represent a variety of clinical manifestations. However, the causes of the syndromes remain unknown. PWS and AS show similar cytological deletions but the opposite parental origin of deletion, i.e., paternal origin in PWS and maternal origin in AS. In addition, uniparental disomy has been found in karyotypically normal PWS patients. Thus, the "genomic imprinting" hypothesis has been proposed that parental genes localized at $15 \mathrm{q} 11$-q12 are differently imprinted in epigenetic process and gene behaviors are different in offspring: a loss of the paternally-derived allele may lead to PWS, while a loss of the maternally-derived allele may result in AS. In this mndel it in ..... ned that the alternative phenotype of PWS would be AS.

IS and 13 AS patients molecular-genetically. DNA deletions were of PWS patients and in $8(61.5 \%)$ of AS patients, using 6 DNA 11-q12 as probes. Excess gene doses were observed in 3 PWS paon two PWS patients confirmed uniparental disomy. Deletion 
ranges differed among patients with either syndrome. Common sites of DNA rearrangements in the patients examined is a segment between D15S9 and D15S12 for PWS, and that between D15S11 and D15S10 for AS, if the 5 loci examined are ordered as cen-D15S18-D15S9-D15S12-D15S11-D15S10-qter. These common sites are not inconsistent with the sites common to all the patients studied by others. Thus, it is likely that the gene loci for the two syndromes are different. RFLP study on an AS family revealed all the 3 affected children have deletions at D15S10 locus, and both of their mother and a maternal grandfather also have deletions at this locus. This finding support the genomic imprinting event that an individual who inherits a deletion from mother have AS phenotype, but does not support the previous model that paternally-derived deletions may result in the PWS phenotype, because the mother of this family is phenotypically normal. This curious finding can be explained by the assumption that the genes for the two syndromes are different.

LGS represents the symptoms of both well-known autosomal dominant disorders, tricho-rhino-phalangeal syndrome type I (TRPS I) and multiple exostoses. LGS patients have deletions at $8 \mathrm{q} 23-\mathrm{q} 24$, while TRPS I patients sometimes show similar deletions. Thus, it is presumed that the difference of deletion ranges would reflect an involvement or noninvolvement of the exostosis gene. With this strategy, we are studying to isolate the exostosis gene. We dissected a 8q23-q24 band region and obtained the band-specific clones. Using the clones as probes, deletion analyses are being performed in patients with either LGS or TRPSI to obtain candidates of the gene.

SII-3. MOLECULAR GENETIC STUDIES OF CHRONIC MYELOGENOUS LEUKEMIA AND DETECTION BY PCR OF A MINIMAL RESIDUAL DISEASE AFTER TREATMENT. Tatsuo ABE, Hikari NISHIGAKI, Johji INAZAWA, Shouhei YOKOTA and Shinichi MISAWA (Dept. Hygiene \& Med., Kyoto Pref. Univ. Med., Kyoto 606)

Using Southern blot hybridization, pulsed field gel electrophoresis and chromosomal in situ hybridization, $\mathrm{Ph}^{1}$ chromosome was precisely analyzed. A total of 32 pts with CML was subjected to the study, in which 23 had a standard, 6 a complex $\mathrm{Ph}^{1}$, and remaining 3 were $\mathrm{Ph}^{\mathrm{I}}(-)$ ve. Six pts with $\mathrm{ALL}$ was also studied. In all 29 pts with $\mathrm{Ph}^{1}$ $(+)$ ve $\mathrm{CML}$, rearrangement had occurred within $5.8 \mathrm{~kb}$ M-bcr. Distribution of breakpoints in pts with complex $\mathrm{Ph}^{1}$ 's did not differ from that in Pts with a standard $\mathrm{Ph}^{1}$ except for some tendency of breakpoints clustered to $5^{\prime}$ region, and alleic deletion within M-bor found in $50 \%$ of cases. On the other hand, all 6 cases with $\mathbf{P h}^{1}(+)$ ve ALL, breakpoints were found in $\mathrm{m}$-bcr. Even in $\mathrm{Ph}^{1}(-)$ ve Pts bcr-abl rearrangement was shown. In addition, mapping study revealed retranslocation after critical junction and/or insertion. Secondly, minimal residual disease (MRD) in CML after BMT was performed by PCR. Residual leukemic cells could successfully detected by this technique, which may provide the precise prognosis of these pis after the treatment.

SII-4. CLINICAL APPLICATION OF RESTRICTION FRAGMENT LENGTH POLYMORPHISMS (RFLP). Yusuke NAKAMURA (Div. Biochem., Cancer Inst., Tokyo 170)

DNA technology based on RFLP (Restriction Fragment Length Polymorphism) has

Vol, 36, No. 1, 1991 
brought a new system to the fields of medicine and forensic science, especially for the study of genetic diseases and for identification of individuals for forensic purposes. Linkage analysis based on segregation of RFLP in affected families has already determined the chromosomal localization of genes that harbor mutations responsible for many genetic diseases, and highly polymorphic VNTR (Variable Number of Tandem Repeat) markers are being used to increase the efficiency of such studies. 